\title{
Design and research of cloud computing resource data monitoring system
}

\author{
Wei Guanghui ${ }^{1 \text {, a }}$ \\ ${ }^{1}$ ChongQing College of Electronic Engineering, computer college,ChongQing,401331,china \\ aqdbiq@163.com
}

Keywords: Cloud computing; Resource data; Monitoring; Design

Abstract. This paper designs the monitoring system of cloud computing center resource, and puts forward the data collection, transmission, storage and viewing of the three levels of Terminal Ordinary nodes, Server Management node and Managers Monitoring center, so as to realize the real-time monitoring of cloud computing resources, including CPU, memory and network resources. The research result is that the scheduling system can fully grasp the computing resources in the cloud computing environment, and provide the data support for the follow-up system resource scheduling.

\section{Introduction}

With the continuous development of virtualization technology in cloud computing, cloud computing data center is also stronger in the change, stop the development and the realization of normal management experience is cloud computer data to guarantee the normal operation, which requires data resources comprehensive monitoring of the running state including all software and hardware and consumption, in order to solve the cloud computing center IT architecture scalability, configurability and other requirements, these characteristics require the computing resource center is based on the resource monitoring in the cloud[1]. Cloud computing resources data monitoring data obtained is to provide data support for cloud computing and dispatch center, through cloud computing resources data monitoring, in the cloud computing task scheduling, load balancing, data center expansion, hardware and software problems timely feedback, Providing views and management data center resources to the cloud computer manager, and ensuring the reliability and availability of cloud computing services.

\section{Monitoring of the object and the realization of the function}

Cloud computing resources to monitor the object: the main memory resources, CPU resources, network resources and its comprehensive application. Including the memory size and memory resource utilization; CPU resources including the number of CPU, CPU and the utilization rate of frequency; cyber source including card speed and effective transmission rate; comprehensive application of operating system includes the use of cloud computing, virtual or physical machine using IP and in the data center.

Cloud computing data monitoring of the realization of the function including data management, information view and forecast analysis. Specifically, the realization of the function is: for a virtual server or to view its utilization rate, the number of processor model, memory, speed of information and the utilization rate of the size, and the operating system, IP address and other information; network topology, view the current cloud computing data center resources including cluster, server and virtual machine information, so as to achieve the scale of network logic changes etc..

\section{Implementation of cloud computing resource data monitoring system}

The key technology of cloud computing resource monitoring data including network communication, PostgreSQL database, Web application development and XML technology. In this paper the research on how to realize the monitoring system, using XML technology. Because XML[2] (Extensible Markup Language) is a common way of data exchange, with the platform, language and system independence, and the advantages of intelligent support code, intelligent search, XML technology has been widely used as cross platform

The form of mutual data, it is very suitable for cloud computing resource data monitoring system requirements.

The document structure of XML document is a tree structure, XML has only one root node, the XML can be read into memory form the tree structure, its advantage is that it can quickly achieve the tree traversal, insert, delete, the disadvantage is the need to read the whole XML, use system resources more, but because the monitoring system the XML file is very small, it is used for data query traversal is still very convenient.

Based on the characteristic of XML document structure tree, tree topology can build cloud computing resources data monitoring system, as shown in figure 1: 


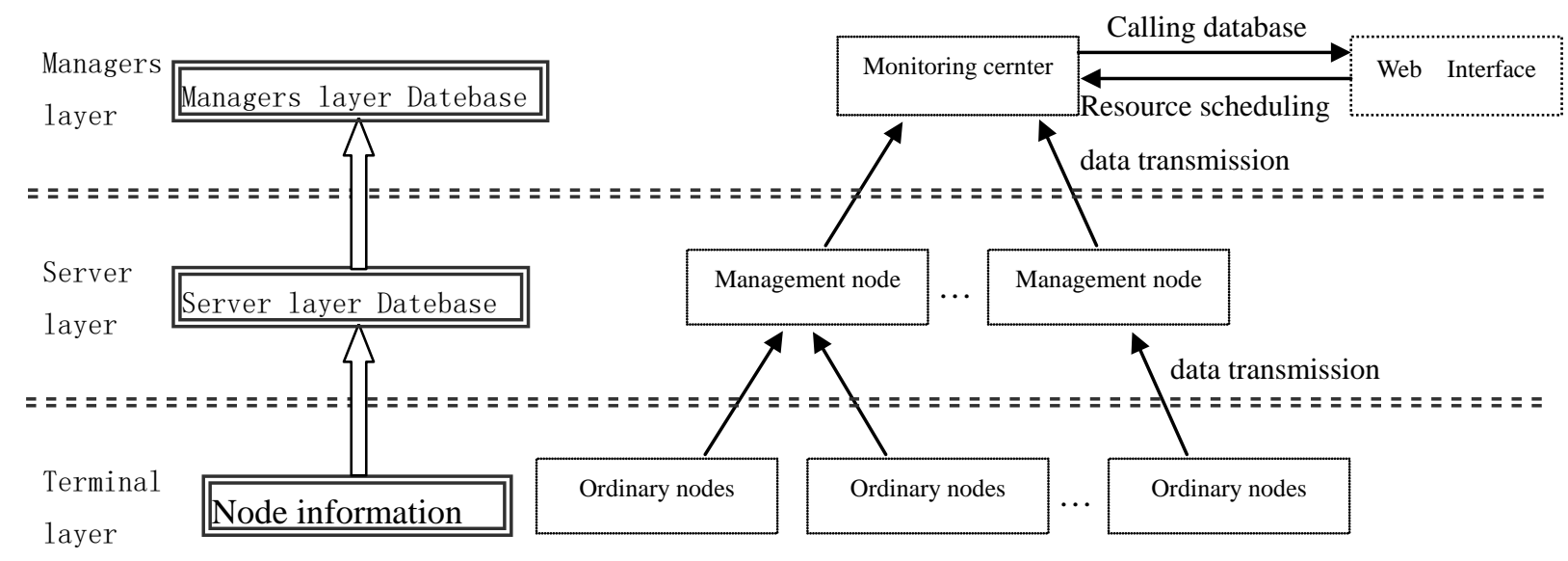

Figure 1. Topology structure of monitoring system

The design requirements of the Terminal of the monitoring system is to obtain the information of a single node, and then to the Server side. Set the XML file on the Terminal side to configure its properties, these properties include: $\mathrm{CPU}$, memory, network properties and their utilization rate, when the Terminal after the start, according to the configuration file to read the information in a fixed time interval preset, and this information to Server.

\section{1 Terminal}

The basic working process of Terminal: Terminal after a successful start-up, pre loading configuration file created, then connect the Server port by Scoket[3], in this process, if the connection is successful, is to obtain relevant information of the machine, send to the Server terminal, if it fails over time after the client restart.

The working process: on the Terminal side, the need to achieve universal for different operating system, information collection methods need to be consistent, we use the Java cross platform, to achieve access to the server status data by calling the Hyperic-hq based package sigar.jar[4]. Sigar.jar package is a local method to call the operating system API to obtain the system related data, including CPU, memory, bandwidth, network information, system information, etc.. In addition, the bottom of the sigar.jar is written in C, by calling the local method to obtain the system related data, the collection of system information can be more convenient to complete the interface provided by API sigar.

Terminal configuration on the XML file, used to find the IP Server address, and then Scoket Terminal connection, to achieve the main function of the code:

Scoket=new Scoket(addr,3233); // Gets the IP address of the server, of which 3233 is the set port number

oos=new ObjectOutStream(socket.getOutputStream());

After the success of the Scoket connection, Terminal first to get their fixed information, that is, the hardware information Server through Scoket to ComputerStaticInfo:

Public class ComputerStaticInfo implements Serializable \{

Private String IP;

Private String CPU;

Private String MEM;

Private String BW;

Private String Mac;

\}

ComputerStaticInfo csi=this.getStaticInfo();

Oos.writeObject(csi);

Then, Terminal again according to every fixed time to get a self utilization information ComputerInfo through Socket sent to the Server:

Public class ComputerInfo implements Serializable \{

Private double CPUUtility;

Private double MemUtility;

Private double BWUtility; 
\}

ComputerInfo ci=new computerInfo();

oos.writeObject(sendInfo();

Thread.slep(t*1000); // t as an integer, according to the monitoring need to set, used to set access to the fixed time interval.

Because the virtual machine users of cloud computing at Terminal, therefore, to monitor the Terminal users also need to monitor the virtual machine, the need to monitor the Terminal of the boot automatically, for the Linux series Terminal, can modify the /.bash_profile file, java_jar client.jar can be increased; in view of the Windows system can pass to the registry to achieve automatic boot operation[5].

\section{2 Server}

Server needs to be collected from the Terminal of the transmission of information to the Managers side, the basic working process is: Server end after the start of loading pre create configuration files, then connect the Managers terminal through Scoket (if the connection fails, the need to modify the configuration file), after a successful connection, waiting for the Terminal side of the Scoket connection, and Terminal after a successful connection, the Terminal sends information received and stored in the local database, and then to encapsulate the information, and transfer to Managers end[6].

Concrete realization:

Establish database server in Server, in order to achieve a large number of terminals connected to the receiving and forwarding the Managers terminal to the Terminal side of the message. In the Server database, the main design of the two tables: FixComputers table (storage of fixed information) and ComputerInfo table (storage and utilization of information), the table structure as shown in Table 1 and table 2 .

Table 1. fixed information

\begin{tabular}{|c|c|c|c|c|}
\hline Field name & type & $\begin{array}{l}\text { Whether } \\
\text { is empty }\end{array}$ & & describe \\
\hline ComputerIP & text & no & & Server IP \\
\hline CPU & text & no & & CPU Info \\
\hline OSName & text & no & & Operating system type \\
\hline Mem & text & no & & Memory size \\
\hline Bandwidth & text & no & & Network bandwidth \\
\hline State & text & no & & Status flag \\
\hline ComputerType & text & no & Physi & al machine or virtual machine \\
\hline \multicolumn{5}{|c|}{ Table 2. Utilization information } \\
\hline Field name & \multicolumn{2}{|c|}{ type } & $\begin{array}{l}\text { Whether is } \\
\text { empty }\end{array}$ & describe \\
\hline ComputerIP & \multicolumn{2}{|c|}{ text } & no & Server IP \\
\hline CPURate & \multicolumn{2}{|c|}{ real } & no & CPU utilization information \\
\hline MemRate & \multicolumn{2}{|c|}{ real } & no & Memory utilization information \\
\hline BandwidthRate & \multicolumn{2}{|c|}{ real } & no & $\begin{array}{c}\text { Bandwidth utilization } \\
\text { information }\end{array}$ \\
\hline Time & \multicolumn{2}{|c|}{$\begin{array}{c}\text { Timestamp } \\
\text { without time } \\
\text { zone }\end{array}$} & no & $\begin{array}{l}\text { The time to obtain the utilization } \\
\text { of the time zone }\end{array}$ \\
\hline
\end{tabular}

Server client will receive the information from the Terminal side, including the fixed part of the information and the use of information into the database, the realization of the Scoket Server code as follows:

ServerSocket ss=new ServerSocket(3233); // Port number and Terminal correspondence

Socket socket=ss.accept();

UseDB usedb=new UseDB();

Usedb.doInsert(“Fixcomputers",restrictions1,resValues1);

In the server side, the need to transfer from the Terminal side of the information (including FixComputers and ComputerInfo) into the database, the following is the ComputerInfo of the storage operation:

Create table”ComputerInfo"

(“ComputerIP” text NOT NULL, 


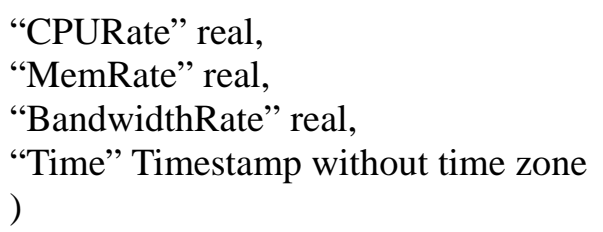

Also need to carry out similar operations on the part of FixComputers, and will not go.

Server end as the intermediate level between terminal and Managers terminal, to provide storage and forwarding function, do not need to deal with a large amount of data, but require the Server terminal to ensure the robustness and stability, therefore, the Server end by a dedicated server to serve, to ensure that the data transmission capability of Server terminal[7].

\section{3 Managers}

On the Managers side also need to be like the Server side, to establish their own database to receive information from the Server forwarded. Its database design is similar to the aforementioned Server side, only to increase the data center description logo[8].

Managers is responsible for the entire cloud computing resources data monitoring, Terminal monitoring information forwarded to the receiving end Server, enables administrators to remotely view the taste of cloud computing resources, user request and server utilization of various information, specific functions include: all Server and cloud in the center of the end of the receiving end information communication, Terminal Server sent to browse all the information network; provide remote services required by the cloud service monitoring system; access to the cloud service scheduling system database, the user access request information, and the user request information is displayed; the Managers terminal according to the user request information, calculate the optimal number of servers required according to the current; using the detected rate estimation of energy consumption, the server. Managers side is an enhanced Server side, receiving information from the Server side, but no need to forward again, in fact, the method can refer to the Server.

\section{Conclusions}

In this paper, through the three levels of design and calculation of monitoring system of resource data to cloud, to the designated information terminal node on the access to the server, and eventually forwarded to the management end, realize the calculation of monitoring resources on cloud data management in the end, so that managers can remotely view, in the management and deployment of cloud computing resources.

\section{References}

[1] Tan Haoyu. Monitoring System in Virtual Machine Management Platform [D]. Huazhong University of Science and Technology:master,2008.

[2]Patel, Chandrakaant D., Cost Model for Planning, Development, and Operation of a Data Center. Internet System and Storage Laboratory, HP Laboratories, Palo Alto, June 9, 2005.

[3] Liu Peng. cloud computing [M]. BeiJing: Publishing House of electronics industry,2010.

[4] WANG Zejun.Design and implementation of Network Monitor and Control System Based on Load Balaneing [D].Guangdong Universith of Technology:master,2008.

[5] YANG Gang,SUI Yu-lei. .Adaptive approach to monitor resource for cloud computing platform[J].Computer Engineering and Applications,2009, 4(29):14-17.

[6] XU Ye,YUAN Min,LI Guojun. Design and Implementation of Remote Monitor and Control System Based on Embedded Web Server[J].JiSuanJi Yu XianDaiHua,2013,2(210):94-98.

[7] Tong Duan,Dong Xiaoshe,Li Jiyun.The Design and Implementation of Web-Based Cluster Remote Monitoring[J]. Computer Engineering and Applications,2003,35:100-102.

[8] Breett McLaughlin. Java and XML[M]. BeiJing: China Power Press,2011. 